DOI: $10.2478 /$ lpts-2018-0020

SOLID STATE PHYSICS

\title{
FINDING ELECTRON-HOLE INTERACTION IN QUANTUM KINETIC FRAMEWORK
}

\author{
E. Klotins \\ Institute of Solid State Physics, University of Latvia, \\ 8 Kengaraga Str., Riga, LV-1063, LATVIA \\ klotins@cfi.lu.lv
}

\begin{abstract}
The article presents a quantum kinetic framework to study interacting quantum systems. Having the constituting model Hamiltonians of two-band semiconductor and the photoexcited electron-hole pair, their quantum kinetic evolution has been revisited. Solution to this nonlinear problem of electron-hole interaction is obtained making use of the self-consistency loop between the densities of photoexcited electrons and holes and the pairwise interaction terms in the constituting model Hamiltonians. In the leading order, this approach supports the required isomorphism between the pairwise interaction and the birth and annihilation operators of the photoexcited electrons and holes as a desirable property. The approach implies the Hilbert space and the tensor product mathematical techniques as an appropriate generalization of the noninteracting electron-hole pair toward several-body systems.
\end{abstract}

Keywords: Hilbert spaces, photoexcited electron-hole pair, quantum electrodynamics

\section{INTRODUCTION}

Quantum kinetic description of mater is a problem at the frontiers of condensed matter physics and constitutes the key challenge to the technological developments based on light-semiconductor interaction. This motivates for a renewed effort to develop a systematic framework for the electronic structure of interacting quantum systems in a manner going beyond the typical weak interaction approaches and available to complex many-body phenomena characterised by coexistence of the constituting particles and fields. Conventional quantum mechanics is successful in the case of particles, which simply propagate with no interactions. However, in the study of quantum many-body problems of realistic complexity the focus of attention is displaced from the one particle kinetics to multi-particle kinetics and gives rise to the quasiparticle solutions. The associated quantum kinetic model of the electron-hole pair is an important field of research and has been widely developed and extensively studied by V. N. Pervushin [1], S.A. Smolyansky [2] and collaborators [3]-[5]. 
The system found implicitly behind these models is a semiconductor at the temperature low enough to confine the charge carriers, the electron and the hole, to the impurities. The impurity concentration is presumably small and obeys conditions of the electron-hole symmetry. The set of parameters adapted in [1]-[5] and categorised here as the fundamental level constituents includes the effective mass of the electron and the hole, their charge, and the value of the main gap between the conduction and valence bands. This free field picture is perturbed by optical radiation appropriate for transition of an electron between the conduction and valence bands. The resulting quantum kinetics of the electron-hole pair obeys the special relativity and symmetry principles in a unitary and non-perturbative quantum description as an advantage.

By revisiting the electron-hole interaction problem, the aim of the present manuscript is to develop the approach of [1]-[5] toward the complex multi-particle systems in terms of interacting fundamental level constituents. In this regard, the manuscript provides an illustrative construction for the electron-hole pair as a compound system constituted of two subsystems, an electron and a hole, whose pairwise interaction comes into the game in time dependent electromagnetic radiation [1]. The required mathematical formalism includes the linear algebra [6] within the oscillator equation framework [7]-[10] as a promising and elegant formalism that relies heavily on the Hilbert spaces concept and underlies the observables of physical objects of interest. In more detail, our concern is the pairwise (Coulomb) interaction between the photoexcited electron and the hole. To proceed, we make use of the quantum electrodynamics [7]-[10] and the quantum kinetics [1], [11] framework.

The novelty, implemented in this framework firstly to the best of author's knowledge, is definition of the combined electron-hole system as the tensor product of the electron and hole states. Within the associated Hilbert space framework, the electron-hole pair comprising two particles $(\mathrm{N}=2)$ is found in accord with [3] as expected. Extensions toward $\mathrm{N}>2$ for constructing quantum mechanical theories of several particle interacting Coulomb systems are available as a desirable property. Another novelty is the self-consistency conditions between the observables as the quantum kinetic result and the fundamental constituents of the constituting macroscopic Hamiltonians.

The physical picture of this framework include spatially homogeneous interacting spineless Coulomb systems under a spatially homogenous time-dependent electric field represented (at appropriate wavelength) by the electromagnetic vector potential in the Coulomb gauge.

The manuscript is organised as follows. Section 2 presents (i) the essential topics used in the prototypical non-interacting electron-hole pair model and (ii) the first steps towards a unifying framework for interacting extended quantum systems with interacting quantum constituents. Special attention is paid to the connection between the constituting macroscopic Hamiltonians and the associated Lagrangian density. Section 3 comprises a formal development of quantum field functions as a prerequisite for subsequent field-particle correspondence going beyond the usual application of wave functions. Special attention is paid to the symmetry and relativistic requirements that force the number of electron not to be conserved under time dependent optical radiation. Section 4 is addressed to the birth and annihilation of 
electron-hole pairs in terms of the birth and annihilation operators with optical radiation and pairwise electron-hole interaction as key entities. Section 5 provides an abstract of the self-consistency loop connecting the quantum field and the quantum kinetic results. Section 6 concludes with a summary of the results and some remarks on future perspectives of other systems of interest.

\section{BASIC CONCEPTS AND DEFINITION OF TERMS}

The section provides a sketch of the elactron-hole pairwise interaction and the many-particle approaches. The constituting Hamiltonian is the following:

$$
H=\frac{1}{2 m}[\mathbf{p}-q \mathbf{A}(\mathbf{x}, t)]^{2}+V,
$$

where $\mathrm{q}$ is the electric charge of a photoexcited particle with momentum $\mathbf{p}$ and $\mathbf{p} 2 /(2 m)$ is regarded to its kinetic energy. In the Dirac's sea treatment, the charge for electron and hole is $q_{e}=-|e|$ and $q_{h}=+|e|$, respectively. The potential energy term $V$ is the bandwidth, constituting the simplest model of an electron-hole pair [2]. The optical radiation involves a spatially homogenous time-dependent electric field represented by the vector potential $\mathbf{A}$ in the Coulomb gauge $\nabla \cdot \mathbf{A}=0$. The electronhole pair is categorised as a complex physical system where both the electron and the hole obey the time-independent free field Schrödinger equation. The physical picture involves the electron and the hole both contributing to the potential energy proportionally to their pairwise interaction.

From the perspective of interacting electron-hole pair, the key entities are basis vectors $\Psi_{c}(\mathbf{x}, t)$ and $\Psi_{v}(\mathbf{x}, t)$ that are elements of the state spaces $S_{c}$ and $S_{v}$ for the electron and the hole. We define the combined electron hole system as the tensor product:

$$
S_{c v}=S_{c} \otimes S_{v}
$$

It means that for each pair of states $\Psi_{c}(\mathbf{x}, t) \in S_{c}$ and $\Psi_{v}(\mathbf{x}, t) \in S_{v}$ there is a combined state space $\left|\Psi_{c}, \Psi_{v}\right\rangle \equiv\left|\Psi_{c}\right\rangle \otimes\left|\Psi_{v}\right\rangle=\left|\Psi_{c}\right\rangle\left|\Psi_{v}\right\rangle \in S_{c v}$ where the hole is in valence state $\left|\Psi_{v}\right\rangle$ and the electron is in the conduction state of $\left|\Psi_{c}\right\rangle$. For the prototypical system of an electron and a hole, the requested tensor product of electron and hole states is the following:

$$
\left[\hat{E}_{c}-\Delta / 2-\varepsilon_{e}(\hat{\mathbf{p}})\right] \otimes\left[\hat{E}_{v}+\Delta / 2+\varepsilon_{h}(\hat{\mathbf{p}})\right] \Psi_{c} \Psi_{v}=0
$$

A desirable property of the tensor product framework is its applicability to many-particle quantum systems formed in $N$ single-particle spaces as $S^{N}=S_{1} \otimes S_{2} \otimes \ldots \otimes S_{N}$. However, the requirements for appropriate algebraic relations between the operators and the abstract Hilbert spaces must be extended by sev- 
eral special features, including the restrictions of special relativity and the symmetry principles essential for constructing quantum kinetic theories of strongly interacting systems. As expected for two-particle systems, the tensor product (3) turns in the oscillator equation for the joint wave function:

$$
\left[\hat{E}-\left(\Delta / 2+W_{c}\right)-\varepsilon_{c}(\hat{\mathbf{P}})\right]\left[\hat{E}+\left(\Delta / 2+W_{v}\right)-\varepsilon_{h}(-\hat{\mathbf{P}})\right] \Psi(\mathbf{x}, t)=0,
$$

where $W_{c}, W_{v}$ specify the unknown yet impact of pairwise electron-hole interaction regarded to the potential energy in (1). The $\Psi(\mathbf{x}, t)$ is the joint electronhole state in (3) and the kinetic dispersion of the conduction and valence states are defined as $\varepsilon_{c}(\hat{\mathbf{P}})=\left(\hat{\mathbf{p}}^{2}+|e| \mathbf{A} \hat{\mathbf{p}}\right) /\left(2 m_{e}\right), \quad \varepsilon_{v}(-\hat{\mathbf{P}})=\left(\hat{\mathbf{p}}^{2}-|e| \mathbf{A} \hat{\mathbf{p}}\right) /\left(2 m_{h}\right)$. We express the energy operator $\hat{E} \rightarrow i \partial_{t}$ and the momentum operator $\hat{\mathbf{p}} \rightarrow-i \nabla$.

The Lagrangian density associated which the oscillator equation implies two complex conjugate field functions $\Psi(\mathbf{x}, t)$ and $\Psi^{*}(\mathbf{x}, t)$

$$
\begin{aligned}
& L(\mathbf{x}, t)=\frac{1}{\Delta} \\
& \left(\begin{array}{l}
\frac{\left(W_{v}-W_{c}-\varepsilon_{c}(\mathbf{P})+\varepsilon_{v}(-\mathbf{P})\right)^{2}}{4} \Psi \Psi^{*}-\frac{\left(W_{c}+W_{v}+\Delta+\varepsilon_{c}(\mathbf{P})+\varepsilon_{v}(-\mathbf{P})\right)^{2}}{4} \Psi \Psi^{*} \\
+\frac{i\left(W_{v}-W_{c}-\varepsilon_{c}(\mathbf{P})+\varepsilon_{v}(-\mathbf{P})\right)}{2}\left(\Psi^{*} \dot{\Psi}-\Psi \dot{\Psi}^{*}\right)+\dot{\Psi}^{*} \dot{\Psi}
\end{array}\right),
\end{aligned}
$$

where the factor $1 / \Delta$ provides necessary dimension balance, and the kinetic dispersions are obtained by replacements $\varepsilon_{c}(\hat{\mathbf{P}}) \leftrightarrow \varepsilon_{c}(\mathbf{P}), \varepsilon_{v}(-\hat{\mathbf{P}}) \leftrightarrow \varepsilon_{v}(-\mathbf{P})$ valid in the case of quadratic isotropic dispersion. The Lagrangian density (5) and the field functions $\Psi(\mathbf{x}, t)$ and $\Psi^{*}(\mathbf{x}, t)$ are the starting points for the unitary part of the unified framework.

\section{NORMALIZATION OF FIELD FUNCTIONS}

In the present section, the Lorentz invariant, relativistic normalized and canonically quantized field functions are constructed. These special features imply four Fourier transformations:

$$
\begin{aligned}
& \Psi(x)=\int d^{4} p(2 \pi)^{-4} \tilde{\Psi}(p) \exp (-i p x) \\
& \Psi^{*}(x)=\int d^{4} p(2 \pi)^{-4} \tilde{\Psi}^{*}(p) \exp (i p x)
\end{aligned}
$$

where properties of the system are implemented in the momentum space fields $\tilde{\Psi}(p)$ and $\tilde{\Psi}^{*}(p)$. The 4-vector scalar product $p x=E t-\mathbf{p x}$ is defined in $\{+,-,-,-\}$ metric signature.

To proceed to the momentum space fields, we make use of the integral from 
to the generalized scaling property [3] as follows:

$$
\int f(E, \mathbf{p}) \delta(g(E, \mathbf{p})) d E=\sum_{i} f\left(E_{i}\right) /\left|g^{\prime}\left(E_{i}\right)\right|
$$

here the $g(E, \mathbf{p})$ function and the field amplitudes $f(E, \mathbf{p})$ are defined as:

$$
\begin{aligned}
& g(E, \mathbf{p})=\left[E-\left(\Delta / 2+W_{c}\right)-\varepsilon_{c}(\mathbf{P})\right]\left[E+\left(\Delta / 2+W_{v}\right)+\varepsilon_{v}(-\mathbf{P})\right], \\
& f(E, \mathbf{p})=\psi(E, \mathbf{p}) \exp (-i E t) .
\end{aligned}
$$

The field functions (6), (7) turn into

$$
\left.\Psi(\mathbf{x}, t)=\int \frac{d \mathbf{p}}{(2 \pi)^{3 / 2}}\left(\begin{array}{l}
\left(\frac{1}{\sqrt{2 \pi}}\right) \frac{1}{\Delta+W_{c}+W_{v}+\varepsilon_{c}(\mathbf{P})+\varepsilon_{v}(-\mathbf{P})} \\
\psi\left(\Delta / 2+W_{c}+\varepsilon_{c}(\mathbf{P})\right) e^{-i\left(\Delta / 2+W_{c}+\varepsilon_{c}(\mathbf{P})\right) t}+ \\
\psi\left(-\Delta / 2-W_{v}-\varepsilon_{v}(-\mathbf{P})\right) e^{i\left(\Delta / 2+W_{v}+\varepsilon_{v}(-\mathbf{P}) t\right.}
\end{array}\right)\right) e^{i \mathbf{p x}}
$$

Finally, the generalized scaling property (8) is obtained as a function of the macroscopic entities:

$$
\begin{aligned}
& \sum_{i} \frac{f\left(E_{i}, \mathbf{p}\right)}{\left|g^{\prime}\left(E_{i}\right)\right|}=\frac{1}{W_{c}+W_{v}+\Delta+\varepsilon_{c}(\mathbf{P})+\varepsilon_{v}(-\mathbf{P})} \\
& \left\{\begin{array}{l}
\psi\left(\Delta / 2+W_{c}+\varepsilon_{c}(\mathbf{P})\right) \exp \left[-i\left(\Delta / 2+W_{c}+\varepsilon_{c}(\mathbf{P})\right)\right]+ \\
\psi\left(-\Delta / 2-W_{v}+\varepsilon_{v}(-\mathbf{P})\right) \exp \left[i\left(\Delta / 2+W_{v}+\varepsilon_{v}(-\mathbf{P})\right)\right]
\end{array}\right\}
\end{aligned}
$$

The direct field functions (6), (7) turn into

$$
\left.\Psi(\mathbf{x}, t)=\int \frac{d \mathbf{p}}{(2 \pi)^{3 / 2}}\left(\begin{array}{l}
\left(\frac{1}{\sqrt{2 \pi}}\right) \frac{1}{\Delta+W_{c}+W_{v}+\varepsilon_{c}(\mathbf{P})+\varepsilon_{v}(-\mathbf{P})} \\
\psi\left(\Delta / 2+W_{c}+\varepsilon_{c}(\mathbf{P})\right) e^{-i\left(\Delta / 2+W_{c}+\varepsilon_{c}(\mathbf{P})\right) t}+ \\
\psi\left(-\Delta / 2-W_{v}-\varepsilon_{v}(-\mathbf{P})\right) e^{i\left(\Delta / 2+W_{v}+\varepsilon_{v}(-\mathbf{P}) t\right.}
\end{array}\right)\right) e^{i \mathbf{p x}}
$$

and the complex conjugate. 


\section{CANONICAL QUANTIZATION}

As the first step toward the canonical quantization, we redefine the unknown yet $\psi$ - functions in (12) in time-independent creation-annihilation amplitudes $\psi\left(\Delta / 2+W_{c}+\varepsilon_{c}(\mathbf{p})\right)=a_{c}(\mathbf{p}), \quad \psi\left(-\Delta / 2-W_{v}-\varepsilon_{v}(-\mathbf{p})\right)=a_{v}(-\mathbf{p}) \quad \psi^{*}\left(\Delta / 2+W_{c}+\right.$ $\left.+\varepsilon_{c}(\mathbf{p})\right)=a_{c}^{*}(\mathbf{p}), \psi^{*}\left(-\Delta / 2-W_{v}-\varepsilon_{v}(-\mathbf{p})\right)=a_{v}^{*}(-\mathbf{p})$. The subsequent box normalization [13] that involves a box of volume $L^{3}$ allows replacing the integral representation of the field functions (13) by sums on the momentum space $\Sigma_{\mathbf{p}}(2 \pi)^{3 / 2} L^{-3}->\int d^{3} \mathbf{p}(2 \pi)^{-3 / 2}$. Another concern is redefinition of the creation-annihilation amplitudes $a(\mathbf{p})$ in operators $a_{\mathbf{p}}$ by relation $a(\mathbf{p})=(L /(2 \pi))^{3 / 2} a_{\mathbf{p}}$ [13].

Finally, the field functions (6), (7) harmonized with the constituting (reference) Hamiltonians are as follows:





The structure of field functions (14), (15) is in accord with [2] obtained by the decomposition of the (unknown yet) wave function into the superposition of plane waves corresponding to electron and hole.

A complementary to (14), (15) approach is developed in [5], which differs in minor details and is not considered in the present manuscript.

Having the field functions and the Lagrangian density (5) the canonical momenta $\pi=\partial L / \partial \dot{\Psi}$ is as follows:

$$
\begin{gathered}
\pi(\mathbf{x}, t)=\frac{i}{\sqrt{\Delta}} \sum_{\mathbf{p}}\left\{\begin{array}{l}
\frac{\sqrt{W_{c}-W_{v}+\Delta+\varepsilon_{c}(\mathbf{P})+\varepsilon_{v}(-\mathbf{P})}}{2 L^{3 / 2}} \\
\left(e^{i t\left(W_{c}+\Delta / 2+\varepsilon_{c}(\mathbf{P})\right)} a_{c}^{\dagger}(\mathbf{p})-e^{-i t\left(W_{v}+\Delta / 2+\varepsilon_{v}(-\mathbf{P})\right)} a_{v}^{\dagger}(-\mathbf{p})\right) e^{-i \mathbf{x p}}
\end{array}\right\} \\
\pi^{*}(\mathbf{x}, t)=\frac{-i}{\sqrt{\Delta}} \sum_{\mathbf{p}}\left\{\begin{array}{l}
\frac{\sqrt{W_{c}+W_{v}+\Delta+\varepsilon_{c}(\mathbf{P})+\varepsilon_{v}(-\mathbf{P})}}{2 L^{3 / 2}} \\
\left(e^{-i t\left(W_{c}+\Delta / 2+\varepsilon_{c}(\mathbf{P})\right)} a_{c}^{+}(\mathbf{p})-e^{i t\left(W_{v}+\Delta / 2+\varepsilon_{v}(-\mathbf{P})\right)} a_{v}^{+}(-\mathbf{p})\right) e^{i \mathbf{x p}}
\end{array}\right\} .
\end{gathered}
$$


Another derived quantity, the Hamiltonian density, obeys the relation

$$
H=\pi \dot{\Psi}+\pi^{*} \dot{\Psi}^{*}-L(\Psi)
$$

In lowest order approximation [3], accepted in the manuscript, the last term in (18) is discarded without loss of generality. Then the total Hamiltonian is as follows:

$$
H_{t o t}=\frac{1}{2 L^{3}} \sum_{\mathbf{p}}\left(\begin{array}{l}
\left(W_{c}+\Delta / 2+\varepsilon_{c}(\mathbf{p})\right) a_{c}(\mathbf{p}) a_{c}^{\dagger}(\mathbf{p})+ \\
\left(W_{v}+\Delta / 2+\varepsilon_{v}(-\mathbf{p})\right) a_{v}(-\mathbf{p}) a_{v}^{\dagger}(-\mathbf{p})
\end{array}\right) \text {, }
$$

where the pairwise interaction is supported by the non-relaxed photoexcited charges after the optical radiation is switched off.

At the next level of approximation, the kinetic momentum is maintained and the creation-annihilation operators become time-dependent as obtained by replacements $\varepsilon_{c, v}( \pm \mathbf{p})->\varepsilon_{c, v}( \pm \mathbf{P})$ and $a_{c, v}( \pm \mathbf{p})->a_{c, v}( \pm \mathbf{P}, t)$. Then the total Hamiltonian (19) turns in the quasiparticle representation:

$$
H_{t o t}(t)=\frac{i}{2 L^{3}}\left(\begin{array}{l}
a_{e, \mathbf{p}}^{\dagger}(t) \dot{a}_{e, \mathbf{p}}(t)-a_{h,-\mathbf{p}}^{\dagger}(t) \dot{a}_{e, \mathbf{p}}(t)+a_{e, \mathbf{p}}^{\dagger}(t) \dot{a}_{h,-\mathbf{p}}(t)-a_{h,-\mathbf{p}}^{\dagger}(t) \dot{a}_{h,-\mathbf{p}}(t) \\
-a_{h,-\mathbf{p}}(t) a_{e, \mathbf{p}}^{\dagger}(t) \lambda(\mathbf{p}, t)+a_{e, \mathbf{p}}(t) a_{h,-\mathbf{p}}^{\dagger}(t) \lambda(\mathbf{p}, t)-a_{e, \mathbf{p}}(t) \dot{a}_{e, \mathbf{p}}^{\dagger}(t)+ \\
a_{h,-\mathbf{p}}(t) \dot{a}_{e, \mathbf{p}}^{\dagger}(t)-a_{e, \mathbf{p}}(t) \dot{a}_{h,-\mathbf{p}}^{\dagger}(t)+a_{h,-\mathbf{p}}(t) \dot{a}_{h,-\mathbf{p}}^{\dagger}(t)
\end{array}\right)
$$

where $\lambda(\mathbf{p}, t)=\dot{\boldsymbol{\Omega}}(\mathbf{p}, t) / \mathbf{\Omega}(\mathbf{p}, t)$ and $\boldsymbol{\Omega}(\mathbf{p}, t)=\sqrt{W_{c}+W_{v}+\Delta+\varepsilon_{c}(\mathbf{P})+\varepsilon_{v}(-\mathbf{P})}$.

The equations of motion for the birth and annihilation operators follow from the minimal action principle:

$$
S=\int d^{4} x\left\{\pi \dot{\Psi}+\pi^{*} \dot{\Psi}^{*}-H_{t o t}(t)\right\}
$$

The operator equations of motion follows after functional derivation which respect to the field amplitudes

$$
\begin{aligned}
& \dot{a}_{e, \mathbf{p}}(t)=\frac{\lambda(\mathbf{p}, t)}{2} a_{h,-\mathbf{p}}(t)-i a_{e, \mathbf{p}}(t)\left(W_{c}+\frac{\Delta}{2}+\varepsilon_{c}(\mathbf{p}, t)\right) \\
& \dot{a}_{e, \mathbf{p}}^{\dagger}(t)=\frac{\lambda(\mathbf{p}, t)}{2} a_{h,-\mathbf{p}}^{\dagger}(t)+i a_{e, \mathbf{p}}^{\dagger}(t)\left(W_{c}+\frac{\Delta}{2}+\varepsilon_{c}(\mathbf{p}, t)\right) \\
& \dot{a}_{h,-\mathbf{p}}(t)=\frac{\lambda(\mathbf{p}, t)}{2} a_{e, \mathbf{p}}(t)+i a_{h,-\mathbf{p}}(t)\left(W_{v}+\frac{\Delta}{2}+\varepsilon_{v}(\mathbf{p}, t)\right)
\end{aligned}
$$




$$
\dot{a}_{h,-\mathbf{p}}^{\dagger}(t)=\frac{\lambda(\mathbf{p}, t)}{2} a_{e, \mathbf{p}}^{\dagger}(t)-i a_{h,-\mathbf{p}}^{\dagger}(t)\left(W_{v}+\frac{\Delta}{2}+\varepsilon_{v}(\mathbf{p}, t)\right)
$$

Here the field amplitudes are redefined by replacements $a(\mathbf{p}, t) \rightarrow a_{\mathbf{p}}(t)$, and the band indices $v / c$ (valence/conduction) are replaced by quasiparticle indices $h / e$. The first terms in (22)-(25) describe creation and annihilation of the electronhole pairs with the amplitude of interband transitions $\lambda(\mathbf{p}, t)$ under action of the optical radiation. The pairvise interaction is condensed in the amplitude of interband transitions (20) and in the last terms written here as the algebraic representation of the Heisenberg commutator $i[H, a(\mathbf{p}, t)]$ [2]. The set of equations (22)-(25) concludes the quantum unitary evolution stage and is appropriate for making contacts with the adiabatic quantum Vlasov description [11].

\section{FROM QUANTUM UNITARY EVOLUTION TO IRREVERSIBLE QUANTUM VLASOV DESCRIPTION}

Kinetic description of the electron-hole pairs is based on the distribution function formalism well accepted in problems of the particle pair production from the vacuum in strong time-dependent laser radiation [11], [7]. For the electron and the hole distribution functions in quasiparticle representation, we follow [2]:

$$
\begin{aligned}
& f_{e, h}(\mathbf{p}, t)=\left\langle 0_{i n}\left|a_{e, h, \mathbf{p}}^{\dagger}(t) a_{e, h, \mathbf{p}}(\mathbf{p}, t)\right| 0_{i n}\right\rangle, \\
& f_{h}(-\mathbf{p}, t)=\left\langle 0_{i n}\left|a_{h,-\mathbf{p}}^{\dagger}(t) a_{h,-\mathbf{p}}(t)\right| 0_{i n}\right\rangle
\end{aligned}
$$

where the charge conservation implies as $f_{e}(\mathbf{p}, t)=f_{h}(-\mathbf{p}, t)$. The quantum Vlasov equations for the fermion distribution functions [1], [2] are as follows:

$$
\dot{f}_{e, h}(\mathbf{p}, t)=\lambda(\mathbf{p}, t) \int_{-\infty}^{t} d t^{\prime} \lambda\left(\mathbf{p}, t^{\prime}\right)\left[1-2 f_{e, h}\left(\mathbf{p}, t^{\prime}\right)\right] \cos \left(2 \theta\left(\mathbf{p}, t, t^{\prime}\right)\right)
$$

where the alternating phase $\theta\left(\mathbf{p}, t, t^{\prime}\right)=\int_{t^{\prime}}^{t} d \tau[\varepsilon(\mathbf{P}(\tau))+\Delta / 2]$. While the amplitude of the interband transitions follows the applied field instantly, the non-Markovian behaviour is maintained, and the kinetics is adiabatic. The connection between the quantum unitary evolution and the distribution function formalism utilizes a selfconsistent collective field created by electrons and holes that depends in a complex way on the distribution functions. The available extensions involve the tensor product framework (3) and the pairwise interaction managed by recursive application of the time-dependent photoexcited particle number density in the adiabatic particle number basis: 


$$
n(t) \equiv \int d \mathbf{p} f(\mathbf{p}, t)
$$

and of the Coulomb interaction treated in the non-relativistic limit:

$$
W \equiv-\frac{e^{2} n(t)}{4 \pi \varepsilon_{0}\left[\mathbf{r}_{e}-\mathbf{r}_{h}\right]} .
$$

Then, finally, the expected self-consistency loop holds between the densities of photoexcited electrons and holes (29), the pairwise interaction (30) and the Hamiltonian (20) where the effective electron-hole distance emerges as a parameter.

\section{CONCLUSIONS}

Based on the Schrödinger type constituting Hamiltonians of two-band semiconductor and the existence of the photoexcited electron-hole pair, we have reconsidered their (first order) interaction in terms of the quantum unitary and the adiabatic Vlasov description. Although not unique, this model involves the prototypical electron-hole pair as a compound system constituted of two subsystems, an electron and a hole, which obeys the special relativity and symmetry principles crucial for quantum mechanical theories of strongly interacting particles.

This implies the Hilbert space of the compound electron-hole system as tensor product of the constituting subsystems, the electron and the hole, as most appropriate generalization of the prototypical electron-hole pair toward several body systems characterised by coexistence of the constituting particles and fields.

The novelty, addressed to bypass the mathematical difficulties inherent in the pairwise (electron-hole) interaction (30) is the self-consistency loop that connects the phoexcited particle number density (29) and the quasiparticle representation obtained as a quantum kinetic result (20). In the leading order, this novelty provides the required isomorphism between the pairwise interaction and the birth and annihilation operators of the photoexcited electrons and holes.

Although the present manuscript has demonstrated the quantum unitary evolution and the adiabatic Vlasov description for the distribution function of a single interacting electron-hole pair, an insight into other situations of interest is possible such as quantized electromagnetic radiation, half-integer spins and the crystal field models that are the areas of further research.

\section{ACKNOWLEDGEMENTS}

The present research has been supported by the Institute of Solid State Physics, the University of Latvia within the framework of National Research Program IMIS2. [Grant numbers VPPI IMIS2, IMIS4]. 


\section{REFERENCES}

1. Pervushin, V.N., Skokov, V.V., Reichel, A.V., Smolyansky, S.A., \& Prozorkevich, A.V. (2005). The kinetic description of vacuum particle creation in the oscillator representation. Int. J. Mod. Phys. A20, 5689-5704. https://doi.org/10.1142/S0217751X05028909

2. Smolyansky, S.A., Tarakanov, A.V., \& Bonitz, M. (2009). Vacuum particle creation: analogy with the Bloch theory in solid state physics. Contrib. Plasma Phys., 49, 575584. DOI 10.1002/ctpp.2009 10058S

3. Smolyansky, S.A., Bonitz, M., \& Tarakanov, A.V. (2010). Strong field generalization of the interband transitions kinetics. Physics of Particles and Nuclei, 41, 1075-11078. DOI 10.1134/S106377961007021X

4. Pervushin, V.N., \& Skokov, V.V. (2006). Kinetic description of fermion production in the oscillator representation. Acta Physica Polonica B, 37, 2587-2600.

5. Friesen, A.V., Prozorkevich, A.V., Smolyansky, S.A., \& Bonitz, M. (2007). Nonperturbative kinetics of electron-hole excitations in strong electric field. Proc. SPIE 6537, Laser Physics and Photonics, Spectroscopy and Molecular Modeling V II, 653701. doi: $10.1117 / 12.754724$

6. Von Neumann, J. (1932/1996). Mathematical foundations of quantum mechanics, Princeton landmarks in mathematics. Princeton University Press. ISBN 978-0-691-02893-4, MR 1435976

7. Fedotov, A.M., Gelfer, E.G., Korolev, K.Yu., \& Smolyansky, S.A. (2011). Kinetic equation approach to pair production by a time-dependent electric field. Physical Review D, 83(2), id. 025011. DOI:10.1103/PhysRevD.83.025011

8. Grib A.A., Mamaev, S.G., \& Mostepanenko, V.M. (1994). Vacuum quantum effects in strong fields [in Russian], Energoatomizdat, M. (1988); English transl., Friedmann Laboratory Publishing, St. Petersburg (1994).

9. Schmidt, S. M., Blaschke, D., Röpke, G., Smolyansky, S.A., Prozorkevich A.V., \& Toneev, V.D. (1998). A quantum kinetic equation for particle production in the Schwinger mechanism. Int. J. Mod. Phys., E7, 709-722. https://doi.org/10.1142/S0218301398000403

10. Schmidt, S.M., Blaschke, D., Röpke, G., Prozorkevich, A.V., Smolyansky, S.A., \& Toneev V.D. (1999). Non-Markovian effects in strong-field pair creation. Phys. Rev. D59, 094005.

11. Blaschke, D.B., Smolyansky, S.A., Panferov, A., \& Juchnowski, L. (2017). Particle production in strong time-dependent fields. arXiv:1704.04147v1 [hep-ph] 28 Mar 2017.

12. Álvarez-Gaumé, L., \& Vázques-Mozo, M.Á. (2012). An invitation to quantum field theory. Lecture Notes in Physics 839. DOI:10.1007/978-3-642-23728-7_2, Berlin: Springer Verlag.

13. Schwabl, F. (2005). Advanced quantum mechanics. Berlin: Springer Verlag. 


\section{ELEKTRONU-CAURUMU MIJIEDARBĪBA \\ KVANTU-KINĒTISKĀ APRAKSTĀ}

\section{E. Klotiņš}

Kopsavilkums

Raksts veltīts mijiedarbojošos kvantu sistēmu studijām divu zonu pusvadītāja modelī, kas ietver elektronu un caurumu sistēmu, pakḷautu pāru tipa mijiedarbībai. Attiecīgais nelineārais uzdevums atrisināts izveidojot pašsaistes cilpu, kura noslēdzas izmantojot unitāru kvantu kinētiku fotoierosināto elektronu un caurumu skaitliskā blīvuma aprakstam un Vlasova vienādojumu neatgriezeniska elektrona-cauruma pāra mijiedarbības makroskopiskam aprakstam. Rezultāts pirmā tuvinājumā nodrošina nepieciešamo izomorfismu starp pāra mijiedarbību un elektrona-cauruma dzimšanas un anihilācijas operatoriem. Aprēksinos ilustrētais Hilberta telpas un tenzoru reizinājuma matemātiskais formālisms ir vispiemērotākais prototipisko divu daļiņu apraksta paplašināšanai vairākdalinnu sistēmām un tā pielietojums, iespējams, arī citās nozīmīgās situācijās, saistītās ar kvantētu elektromagnētisko starojumu, pus-veseliem spiniem un kristāla lauka model̦iem.

25.03.2018. 